https://www.amerabra.org; https://fspu.uitm.edu.my/cebs; https://www.emasemasresources.com 9th Asia Pacific International Conference on Environment-Behaviour Studies, Faculty of Architecture, Ulisboa, Lisbon, Portugal, 03-04 Jul 2019

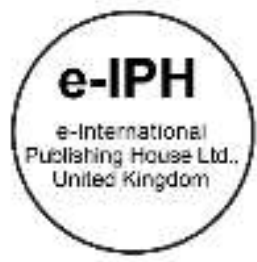

\title{
Anxiety, Depression and Behaviour Problems among Preadolescent Primary School Children
}

\author{
Zamzaliza Abd. Mulud, Norhidayu Sukardi, Norfidah Mohamad \\ Centre for Nursing Studies, Faculty of Health Sciences, \\ Universiti Teknologi MARA, Malaysia
}

zamzaliza@uitm.edu.my, norhidayusukardi88@gmail.com, norfidah@uitm.edu.my Tel: +60332584305

\begin{abstract}
Although are many previous studies reported the factors associated with anxiety, depression and behaviour problems, but few studies have focused on the association between mental health (anxiety and depression) and problem behaviours in children. The current study aimed to determine the association between anxiety, depression and behaviour problems among preadolescent school children $(n=386)$. Results found that $7.7 \%$ and $10.1 \%$ of participants reported abnormal levels of depression and anxiety and it is correlated significantly with the children's problematic behaviours. The findings of the study provide valuable insight into understanding the mental health and behaviours of preadolescence primary school children in Malaysia.

eISSN: 2398-4287 @ 2019. The Authors. Published for AMER ABRA cE-Bs by e-International Publishing House, Ltd., UK. This is an open access article under the CC BYNC ND license (http://creativecommons. org/licenses/by-nc-nd/4.0). Peer-review under responsibility of AMER (Association of Malaysian Environment-Behaviour Researchers), ABRA (Association of Behavioural Researchers on Asians) and cE-Bs (Centre for Environment-Behaviour Studies), Faculty of Architecture, Planning \& Surveying, Universiti Teknologi MARA, Malaysia.

DOI: https://doi.org/10.21834/e-bpj.v4i11.1724
\end{abstract}

\subsection{Introduction}

Currently, mental health problems have become a serious issue, and the related problems are alarming in Malaysia. A report by the World Health Organization estimates that about 10 to $20 \%$ of children worldwide have a mental or behavioural problem (World Health Organization, 2016). Mental health problems that can occur in children include depression, self-harm, generalized anxiety disorder (GAD), post-traumatic stress disorder (PTSD) and eating disorders (Mental Health Foundation, 2015).

Besides, the increasing number of children and adolescents participating in high-risk behaviours such as substance abuse, premarital pregnancy and problem behaviours such as bullying and smoking also occurs concurrently. Worldwide, it has been proven that smoking, oral tobacco use, alcohol and illicit drugs are part of the global health risk (World Health Organization, 2009). In Malaysia, the total number of mental health problems among children 5 to 15 years old were 612 cases (12.1\%) with the highest number of cases was in Selangor which was 87 cases (13.7\%) (Institute for Public Health, 2015). Besides that, the common problem behaviours among children in Malaysia includes property-related crime, drug usage, people-related crime and others. Besides, there was a case where school children as young as 13 years of age had involved in psychoactive drugs (AADK, 2016).

Children often experience emotional turmoil as their minds and bodies develop during the period between childhood and the onset of puberty, which usually designated as between the ages of 10 to 12 in girls and 11 to 13 in boys. During this period, growing up and accepting own self was an important part. However, some young people find it challenging to make this transition to adulthood and may experiment with alcohol, drugs or other substances that can affect their mental and physical health. Therefore, it is essential for early identification of children who at risk for mental health problems as it was the crucial step in establishing prevention or intervention programs. Thus, this current study was conducted to determine the association between anxiety, depression and problematic behaviours among preadolescent primary school children in Selangor, Malaysia.

eISSN: 2398-4287 ( 2019. The Authors. Published for AMER ABRA cE-Bs by e-International Publishing House, Ltd., UK. This is an open access article under the CC BYNCND license (http://creativecommons.org/licenses/by-nc-nd/4.0/). Peer-review under responsibility of AMER (Association of Malaysian Environment-Behaviour Researchers), ABRA (Association of Behavioural Researchers on Asians) and cE-Bs (Centre for Environment-Behaviour Studies), Faculty of Architecture, Planning \& Surveying, Universiti Teknologi MARA, Malaysia.

DOI: https://doi.org/10.21834/e-bpj.v4i11.1724 


\subsection{Literature Review}

World Health Organization (WHO), 2016 estimates that about 10 to $20 \%$ of children worldwide have mental or behavioural problems. Half of these illnesses begin at the age of 14 and three-quarters by mid-20s. In Malaysia, Ahmad, MuhdYusoff, Ratnasingam et al., (2015) has conducted a study to look at the trends of mental health problems among children and adolescents based on three population-based surveys over 15 years. This study found that the prevalence of mental health problems among children and adolescents aged 5 through 15 years showed an increasing trend from $13 \%$ in 1196 to $19.4 \%$ in 2006 and 20\% in 2011. Besides, Essex, Kraemer, Slattery et al., (2009) has conducted a study among 328 children in the USA who had a complete mother and teacher reports of children's symptom since in kindergarten until grades 1, 3 and 5 . This study found that surprisingly, as evidenced by even though only ten kindergarteners evidenced Comorbid Symptoms, $100 \%$ of them developed a pattern of Recurrent Comorbid Symptoms. Furthermore, in Germany, a study conducted by Zach et al., (2016) to identify the association of sociodemographic and environmental factors with the mental health status among preschool children found that $11.0 \%$ of preschool children having mental health problems. Therefore, these situations can be evidence where mental health problems are quite evident as early as kindergarten and alarming worldwide.

Unfortunately, mental health problems persist through childhood and adulthood as they can co-occur or go unnoticed. In Malaysia, a study by Ching, Jiar and Jaffri (2015) was designed to investigate the current level of mental health status in Year Two and Year Three children to identify the early sign of mental health difficulties. This study had involved 5 Chinese medium national-type primary schools located in a suburban area in Johor, with a total of 123 participants. This study showed abnormal total difficulties in nearly $50 \%$ of children from Year Two and Year Three, which mean that these children were suspected of having a high risk for mental health difficulties. Also, a study conducted among 11 to 16 years old students in urban schools in India showed that $10.4 \%$ of participants involved had abnormal total difficulties score, which would suggest that they were also likely to suffer from some degree of mental health issues (Bharath Kumar Reddy, Biswas, \& Rao, 2011).

Many studies were conducted among young adolescent aged 13 to 17 as at this stage they experienced changes either physically or emotionally, and depression was one of the most common mental health problems that can occur among them. Ibrahim et al. (2017) have conducted a study which is to discover the prevalence and predictors of depression and suicidal ideation among adolescents attending government secondary schools in Malaysia. This study found that $32.7 \%$ of students were found to have depression with age group, bullyvictims, smoking, low self-esteem and bullying as factors associated with it. Besides that, a study to determine the prevalence and correlates of depression among adolescents in Malaysia found that $17.7 \%$ of respondents aged 13 to 17 years old had depressive symptoms with feeling lonely, Indian ethnicity, using drugs and being bullied were associated with it (Kaur et al., 2014).

Furthermore, a study conducted by Ramli, Adlina, Suthatar et al., (2008) among secondary school students in Malaysian Community found the prevalence of depression was $10.3 \%$ with being female, Chinese, parent's with low education level and alcohol abuse were factors that associated with depression. Besides, a study by Kawafha (2017) to determine the factors of depression among school children aged 10 to 15 years found that the prevalence of depression was $9.5 \%$ with slightly higher among girls compared to boys. Instead of gender, age, living arrangements and domestic violence were also associated with childhood depression. Therefore, these studies proved that depression commonly occurred in secondary school students and most common factors that associated with it were either related to sociodemographic characteristics such as age, gender and ethnicity or psychosocial factors such as feeling lonely, low self-esteem, being bullied, smoking, using drugs and domestic violence. Thus, this current study was conducted to determine the association between anxiety, depression and problem behaviours among preadolescent school children.

\subsection{Methodology}

\subsection{Study Design}

We conducted a cross-sectional study to collect data regarding anxiety, depression and problem behaviours among preadolescent school children. In this research, the selected population was primary school children aged 10 and 11 years old in the primary schools in Selangor, Malaysia. This population was selected because this is when the children often experience emotional turmoil during the period between childhood and the onset of puberty, which usually designated as between 10 to 12 years old generally

\subsection{Sample Size and Sampling technique}

The sample size was determined by using a sample size calculator by Raosoft Inc. As the population size of primary school children aged 10 and 11 years old was unsure, therefore 20,000 have been used with the margin of error was $5 \%$, level of confidence of $95 \%$ and response distribution of $50 \%$. Thus, the required sample size for this research was 377 .

Multistage sampling technique was used. A list of primary schools was obtained from the State Education Department of Selangor (JPNS). Based on the record, the primary school were categorized according to the district. After that, the schools were further classified into the type of school either regular national type school, integration religious school, Chinese-medium national type school or Tamilmedium national type school. Then, schools randomly selected from regular national school until the targeted sample size achieved. The primary school were selected from Selangor because of the high number of mental or behavioural problems, and obesity problems were in Selangor (Institute for Public Health, 2015).

Sample design used was disproportionate stratified sampling, which unequal distribution of the sample was selected. The uneven distribution of the numbers is considered more appropriate, and representative as the number of children in some primary schools is too large, while others are too small. Thus, a more significant number of respondents will be obtained from larger schools and vice versa. 


\subsection{Data Collection Procedure}

A consent form was given to the parents for the involvement of their children in this research. Then, on the day of data collection, consent forms from the parent were collected from the participants. Children without permission from parents were excluded from participating. After that, the questionnaire was completed by participants in their classrooms with guidance or supervision by investigators. The process of filling the questionnaire took about 20 to 30 minutes, and the questionnaires were returned on the same day study conducted.

A set of self-administered questionnaires was designed to collect the data. The questionnaire consisted of three sections which label as part A, B and C. Self-rated or self-report technique were chosen to answer the questionnaire.

The Malay version of the Strength and Difficulties Questionnaire (SDQ) was used to screen children for emotional and behavioural problems. This questionnaire consists of 25 items which divided into five scales with five items each in one scale. The subscales are conduct problems, hyperactivity problems, emotional symptoms, peer problems and prosocial behaviour. All items in this questionnaire were scored 0,1 or 2 with 0 represent not true, 1 represents somewhat true, and 2 represent certainly true. The resulting score can range from 0 to 40. Self-rated SDQ has good internal consistency with Cronbach's alpha of 0.661, respectively (Zakaria \& Yaacob, 2008).

Anxiety and depression among children were measured using the Revised Child Anxiety and Depression Scale (RCADS). This scale consists of 47 items with 5-point Likert scale responses ranging from never (0), sometimes (1), often (2) and always (3). The total score ranged from 0-141. They produce overall anxiety, and depression score and separate scores for each of the follow sub-scales: separation anxiety; social phobia; generalised anxiety; panic; obsessive-compulsive; total anxiety; and, depression. Both the subscales and the scale have excellent reliability (Chorpita et al., 2005)

\subsection{Data analysis}

Data analysis was computed by using SPSS Version 23.0. Descriptive statistic was used to describe the characteristic of preadolescent schoolchildren initially. After that, the inferential statistic was used to evaluate the data. At the start, a bivariate correlation, which is the Pearson Correlation, was performed to investigate the association of the relationship between each independent variable and the dependent variable separately. The association was considered statistically significant when the $p$-value was at or less than 0.05 ( $p \leq$ 0.05). Finally, differences between continuous variables also were tested. The independent t-test was used to compare the mean score of continuous variables with only two categories such as gender, age, race, medical health status, smoking status, parent's job status and family income. While one-way analysis of variance (ANOVA) was used to compare the mean score of continuous variables with more than two categories, such as the number of siblings.

\subsection{Ethical considerations}

Ethical approval from Universiti Teknologi MARA (UiTM) Research Ethics Committee has been obtained (Referral number: 600-IRMI $(5 / 1 / 6)$ or REC/335/17). Besides, because of the involvement of school children, approval from the Ministry of Education, Malaysia also have been gained (Referral number: KPM.600-3/2/3 Jld 54 (26).

\subsection{Findings}

\subsection{Sociodemographic Characteristics}

A total of 386 participants involved with $189(49 \%)$ were boys, and $197(51 \%)$ were girls, $198(51.3 \%)$ were ten years old, and $188(48.7 \%)$ were 11 years old. Ethnic distribution as for Malay and Indian were $372(96.4 \%)$ and $14(3.6 \%)$. The number of siblings was ranging from 1 to 12, which have been divided into three groups (Group 1: Single; Group 2: 2 to 4; Group 3: 5 and above). The distribution as for Group 1 until Group 3 were $263(68.1 \%), 117(30.3 \%), 6$ (1.6\%), respectively.

Among the participants, $56(14.5 \%)$ of them having medical health problems such as asthma and heart problems, $330(85.5 \%)$ not having any medical health problems and none of them having mental health problems. From all participants, $11(2.8 \%)$ of them were smoking while the remaining $375(97.2 \%)$ were not smoking.

For parent's job status, this variable has been divided into three groups (Group 1: Both are working; Group 2: Either one is working; Group 3: Both are not working). The distribution as for Group 1 until Group 3 were $165(42.7 \%), 221(57.3 \%)$ and $0(0.0 \%)$, respectively. Whereas, for family income per month, low family income, which is less than RM3000 has a percentage of $63.7 \%$ while high family income, which is RM 3000 and above have the percentage of $36.3 \%$.

\subsection{Participants' level of anxiety and depression}

Results found that $30(7.7 \%)$ and $39(10.1 \%)$ of participants reported abnormal levels of depression and anxiety, respectively.

4.3 The association between sociodemographic characteristics and levels of anxiety and depression.

Comparison of mental health status according to sociodemographic characteristics, were investigated by using a t-test and ANOVA (Table1). Among the factors that significantly associated with anxiety and depression are gender $(t-2.17, p=0.01)$ and having a medical illness $(t-1.98, p=0.04)$. 
Table 1 Association between sociodemographic characteristics and total score of anxiety and depression.

\begin{tabular}{|c|c|c|c|}
\hline & Mean (SD) & $t / F$ & $P$ \\
\hline \multicolumn{4}{|l|}{ Gender } \\
\hline Male & 33.22 & 2.17 & $0.01^{*}$ \\
\hline Female & 45.02 & & \\
\hline \multicolumn{4}{|l|}{ Number of siblings } \\
\hline 1 to 4 & 38.66 (18.34) & 0.96 & 0.90 \\
\hline 5 to 8 & $39.56(18.25)$ & & \\
\hline 9 to 12 & $39.00(24.71)$ & & \\
\hline \multicolumn{4}{|l|}{ Family income } \\
\hline Less RM3000 & $38.26(19.16)$ & 0.93 & 0.40 \\
\hline RM3000 to RM5000 & 41.07 (15.91) & & \\
\hline More RM5000 & $37.69(19.30)$ & & \\
\hline \multicolumn{4}{|c|}{ Medical health problems } \\
\hline Yes & $43.43(16.23)$ & 1.98 & $0.04^{*}$ \\
\hline No & $38.19(18.64)$ & & \\
\hline \multicolumn{4}{|l|}{ Smoking status } \\
\hline Yes & $45.36(15.49)$ & 1.18 & 0.24 \\
\hline No & $38.75(18.44)$ & & \\
\hline
\end{tabular}

The relationship between the anxiety, depression, emotional and behavioural problems was investigated using the Pearson correlation coefficient. Results suggested that there was a weak, positive correlation between the anxiety, depression and total difficulty score $(r=$ $0.38, p<0.01$ ), with increases in anxiety and depression associated with higher levels of emotional and behavioural problems.

Table 2 Correlation between anxiety, depression, emotional and behavioural problems

\begin{tabular}{|c|c|c|c|c|c|c|c|}
\hline & 1 & 2 & 3 & 4 & 5 & 6 & 7 \\
\hline 1. Anxiety and Depression & 1 & & & & & & \\
\hline 2. Total Difficulty Score & $0.38^{\star *}$ & 1 & & & & & \\
\hline 3. Prosocial & $0.24^{* *}$ & -0.04 & 1 & & & & \\
\hline 4. Hyperactivity & $0.16^{* *}$ & $0.70^{* *}$ & -0.08 & 1 & & & \\
\hline 5. Emotional & 0.48 & $0.71^{* *}$ & $0.17^{\star *}$ & $0.33^{* *}$ & 1 & & \\
\hline 6. Conduct problems & $0.24^{\star *}$ & $0.73^{* *}$ & $-0.11^{*}$ & $0.40^{\star *}$ & $0.30^{* *}$ & 1 & \\
\hline 7. Peer roblems & 0.10 & $0.55^{* *}$ & $-0.14^{*}$ & $0.12^{*}$ & $0.10^{*}$ & $0.23^{* *}$ & 1 \\
\hline
\end{tabular}

\subsection{Discussion}

For mental health status, there was a significant difference in anxiety and depression with medical health problems. This difference means that, having a medical health problem was significantly at risk of getting mental health problems. This finding is supported by Hysing, Elgen, Gillberg, Lie, \& Lundervold, (2007) where they also found that children with chronic illness (Cl) showed an increased rate of emotional, hyperactivity and peer problems compared to children without $\mathrm{Cl}$. It is also known that illness was the remained predictor that can contribute to mental health difficulties (Kashala, Elgen, Sommerfelt, \& Tylleskar, 2005).

Besides, there was a significant difference in anxiety and depression with gender. The current study found that female students show high levels of anxiety and depression compared to their male counterpart than girls. However, Rimal \& Pokharel, 2013 found that gender difference was not statistically significant in the domain of conduct problems. They revealed that gender difference was statistically significant in emotional problems and total difficulties score. The possible explanation is because male students may express and handle their stress differently than female, adopting a 'problem-focused' rather than 'emotion-focused' coping style. Emotion-focused coping style refers to strategies aimed at reducing psychological distress by avoiding the stress without trying to adjust the situation (Lazarus et al., 1984). The unresolved stress eventually leads to the development of anxiety and depression among female children.

Findings revealed that there was a positive correlation between anxiety and depression with emotional and behavioural problems. The significant relationship that has emerged between these variables in this study suggested that behaviour problems depend on the levels of anxiety and depression of the children. This finding was consistent with research findings obtained from Singh et at (2015) among adolescent in India. Children's mental health status has repeatedly been shown as being related to social competence, skilled social behaviour, pro-social behaviour, and cognition. Children, who typically display fear, anxiety, and withdrawal from social activities commonly demonstrate difficulty when confronted with a range of stimuli, including people, objects, and situations. Thus the emotional and behavioural problems are more prevalent among this population. 


\subsection{Conclusion \& Recommendations}

Nowadays, there was an increasing number of mental and physical health problems not only among adolescent but also among children. Even though various actions have been implemented in preventing these problems, but the effectiveness of these programmes still not achieved the target as many school children still tangled in mental and physical health problems. Therefore, this study has been conducted to determine the association between mental health status and behaviour problems among preadolescent primary school children in Selangor. The result from this study is believed to bring benefits to various stakeholders include other researchers, government, parents as well as to school children in Malaysia. More effective programmes and interventions for school children will be developed and implemented in this country to prevent mental and physical health disorders in children and youth.

Further research in exploring the overall physical health status and the association between physical health and mental health needs to be carried out by using other types of study design. Thus the causal relationship can be determined, using larger samples from other schools with an almost equal number of participants in term of age, gender, race and other sociodemographic data so that the finding can be generalized. Also, to include the psychological aspects based on the biopsychosocial model so that holistic research in exploring the health status of school children can be done. Besides, there is also a need for further study in developing specific physical and mental health intervention programmes instead of health promotional activities for school children.

\section{Acknowledgements}

The authors also like to thank the Universiti Teknologi MARA, Malaysia, for the support of the study. The Ministry of Education supported this work, Malaysia, under the Fundamental Research Grant Scheme (FRGS) project -FRGS/1/2016/SKK07/UITM/02/1.

\section{References}

AADK. (2016). Maklumat Dadah 2016. Report Dadah 2016, 385-386

Ahmad, N., MuhdYusoff, F., Ratnasingam, S., Mohamed, F., Nasir, N. H., MohdSallehuddin, S., ... Aris, T. (2015). Trends and Factors Associated with Mental Health Problems among Children and Adolescents in Malaysia. International Journal of Culture and Mental Health, 8(2), 125-136. https://doi.org/10.1080/17542863.2014.907326

Bharath Kumar Reddy, K. ., Biswas, A., \& Rao, H. (2011). Assessment Of Mental Health Of Indian Adolescents Studying In Urban Schools. Malaysian Journal of Paediatrics and Child Health, 17(2). Retrieved from http://mjpch.com/index.php/mjpch/article/view/276

Ching, L. M., Jiar, Y. K., \& Jaffri, H. (2015). Assessing Mental Health Status among Primary School Children. Advances in Social Sciences Research Journal, 2(3), 228241. https://doi.org/10.14738/assrj.23.1014

Chorpita BF, Moffitt C, Gray J. (2005). Psychometric properties of the revised child anxiety and depression scale in a clinical sample. Behaviour Research and Therapy. 43:309-322. doi: 10.1016/j.brat.2004.02.004

Essex, M. J., Kraemer, H. C., Slattery, M. J., Burk, L. R., Thomas Boyce, W., Woodward, H. R., \& Kupfer, D. J. (2009). Screening for childhood mental health problems: outcomes and early identification. The Journal of Child Psychology and Psychiatry, 50(5), 562-570. https://doi.org/10.1111/j.1469-7610.2008.02015.x

Hysing, M., Elgen, I., Gillberg, C., Lie, S. A., \& Lundervold, A. J. (2007). Chronic physical illness and mental health in children. Results from a large-scale population study. Journal of Child Psychology and Psychiatry, 48(8), 785-792. https://doi.org/doi:10.1111/j.1469-7610.2007.01755.x

Institute for Public Health. (2015). National Health and Morbidity Survey 2015 (NHMS 2015). Vol. II: Non-Communicable Diseases, Risk Factors \& Other Health Problems. Ministry of health (Vol. II). https://doi.org/10.1017/CBO9781107415324.004

Kashala, E., Elgen, I., Sommerfelt, K., \& Tylleskar, T. (2005). Teacher ratings of mental health among school children in Kinshasa, Democratic Republic of Congo. European Child \& Adolescent Psychiatry, 14(4), 208-215. https://doi.org/10.1007/s00787-005-0446-y

Kaur, J., Cheong, S. M., Mahadir Naidu, B., Kaur, G., Manickam, M. A., Mat Noor, M., ... Rosman, A. (2014). Prevalence and Correlates of Depression Among Adolescents in Malaysia. Asia-Pacific Journal of Public Health, 26(5S), 53S-62S. https://doi.org/10.1177/1010539514544356

Kawafha, M. (2017). Increasing Factors of Depression among School Children Aged 10-15 years. Gazi Medical Journal, 28(1), 11-16. https://doi.org/10.12996/gmj.2017.04

Lazarus, R. S., \& Folkman, S. (1984). Stress, appraisal, and coping. New York: Springer Pub. Co.

Mental Health Foundation. (2015). Children and young people. Retrieved from https://www.mentalhealth.org.uk/a-to-z/c/children-and-young-people

Mental Health Foundation. (2018). Physical Health and Mental Health. Retrieved from https://www.mentalhealth.org.uk/a-to-z/p/physical-health-and-mental-health

Ramli, M., Adlina, S., Suthahar, A., AB, E., FM, A., AHH, N., ... Karuthan, C. (2008). Depression among Secondary School Students: a Comparison between Urban and Rural Populations in a Malaysian Community. Hong Kong Journal of Psychiatry, 18(2), 55-61. Retrieved from http://search.ebscohost.com/login.aspx?direct=true\&db=jh\&AN=105794509\&site=ehost-live

Singh, K., Junnarkar, M., \& Sharma, S. (2015b). Anxiety, stress, depression \& psycho-social functioning of Indian adolescents. Indian Journal of Psychiatry, 57(4), 41-48.

World Health Organization. (2016). Mental Health-Child and adolescent mental health. Retrieved from https://www.who.int/mental_health/maternalchild/child_adolescent/en/ 
World Health Organization. (2009). Global Health Risks: Mortality and burden of disease attributable to selected major risks. Bulletin of the World Health Organization, 87, 1-70. https://doi.org/10.2471/BLT.09.070565

Zach, A., Meyer, N., Hendrowarsito, L., Kolb, S., Bolte, G., Nennstiel-Ratzel, U., ... Liebl, B. (2016). Association of sociodemographic and environmental factors with the mental health status among preschool children-Results from a cross-sectional study in Bavaria, Germany. International Journal of Hygiene and Environmental Health, 219(4-5), 458-467. https://doi.org/10.1016/j.jiheh.2016.04.012

Zakaria, Z., \& Yaacob, M. J. (2008). Psychiatric morbidity among children and adolescents living in orphanages, Kota Bharu, Malaysia. International Medical Journal 15(3), 183-188. 